\title{
Effects of Side Walls on Pipe Inlet Flow (Drag Reduction by Separated Flow Control Using Ring Shaped Small Obstacle)*
}

\author{
Toshitake ANDO $^{* *}$, Toshihiko SHAKOUCHI ${ }^{* *}$, Hiroyuki YAMAMOTO** and \\ Koichi TSUJIMOTO** \\ ** Graduate School of Engineering, Mie University \\ Kurimamachiya-cho 1577, Tsu-shi, Mie 514-8507, Japan \\ E-mail: shako@mach.mie-u.ac.jp
}

\begin{abstract}
The flow from a wide space into a pipe has a large annular separated vortex region just after the inlet corner. This vortex region produces large flow resistance, or drag, in this kind of flow. To reduce the drag, a means to control flow in order to suppress the vortex region is needed. In this study, a simple method to reduce the drag of the pipe inlet flow by mounting a small ring-shaped obstacle instead of a bell-mouth has been proposed and examined. The effects of the side-walls and their offset distance on drag reduction were also examined. The small offset distance corresponds to the case in which the pipe inlet is placed near the bottom or corner of the tank. The distributions of pressure and velocity components in the axial direction at several cross-sections were measured, and a visualized flow pattern of the water flow just after the pipe inlet was examined. The effects of the small ring-shaped obstacle on drag reduction were also examined. It was clarified that the inlet loss (drag) coefficient was reduced by a maximum of about 90 percent by mounting the ring-shaped obstacle.
\end{abstract}

Key words : Pipe Inlet Flow, Flow Separation, Flow Control, Drag Reduction, LDA

\section{Introduction}

The flow from a wide space (for example, a reservoir or water storage tank) into a pipe has a large annular separated vortex region just after the inlet corner [only half of the radius region is shown in Fig.1 (a), because of symmetry]. This kind of flow is frequently seen in various types of industrial facilities or equipment.

The vortex region, in which the pressure is lower than the surrounding area, at the pipe inlet causes considerable drag of the pipe inlet flow and is usually suppressed using a bell-

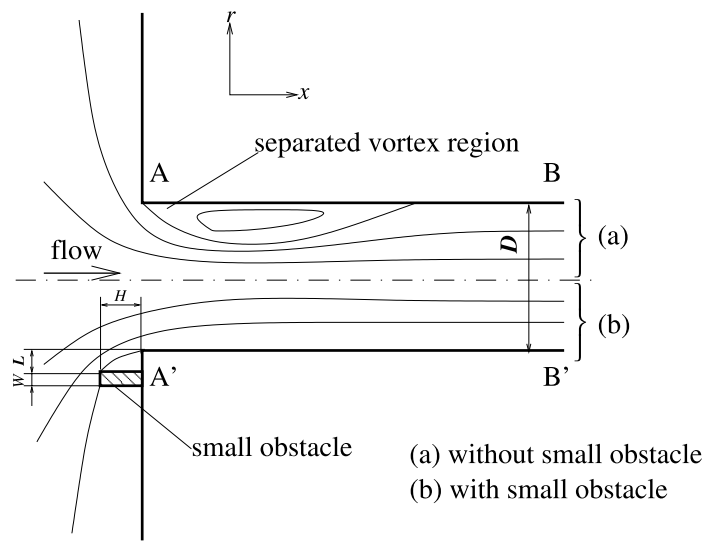

Fig. 1 Flow model of pipe inlet flow

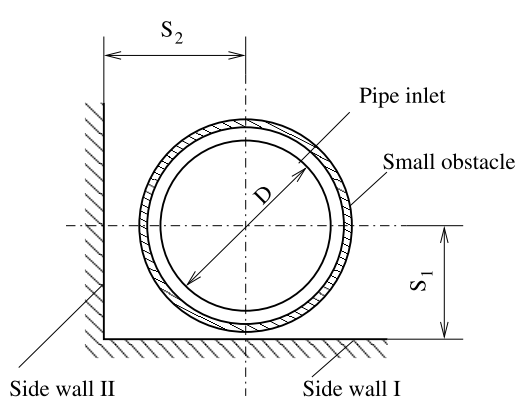

Fig. 2 Side walls 
mouth. However, a bell-mouth is very expensive when used in a large-scale facility that is built with an inlet corner.

The pipe inlet flow is a particular type of flow in an abrupt contraction pipe. It corresponds to the case in which the cross-sectional area of an upstream pipe is infinite. Additionally, an abrupt contraction pipe is an axisymmetric case of a forward-facing step. Many studies have been done on the forward-facing step and two-dimensional abrupt contraction or abrupt contraction pipe flows. For example, Tropea and Gackstatter ${ }^{(1)}$ clarified the flow characteristics over an obstacle on a flat plate by obtaining detailed measurements. Kiya and Nozawa ${ }^{(2)}$ and Taulbee and Robertson ${ }^{(3)}$ studied the flow around a blunt cylinder and forward-facing step, respectively. Liou and $\mathrm{Kao}^{(4)}$ and Chiang ${ }^{(5)}$ numerically analyzed a two-dimensional abrupt contraction flow.

Fukunishi, et al. ${ }^{(6)}$ proposed a method to control the vortex on the upper step surface of a forward-facing step by using a small vibration of the step corner. In the past, the authors described a method to control and suppress the vortex that was formed on the upper step surface of a forward-facing step by mounting a small obstacle on the lower step surface ${ }^{(7)}$. Furthermore, the authors demonstrated that the vortex region on the downstream pipe wall of an abrupt contraction pipe is suppressed and drag is reduced by mounting a small ring-shaped obstacle on the wall of the upstream pipe ${ }^{(8)}$.

The purpose of this study was to clarify that the vortex region on the pipe wall at the inlet can be controlled and suppressed, and that the inlet loss or drag can be reduced by mounting a small ring-shaped obstacle on the reservoir wall, as shown in Fig.1(b).

In actual facilities, the pipe inlet is near the bottom or side wall or the corner of the tank, as shown in Fig.2. We also examined the effects of the side walls on drag reduction. The small offset distances of the side walls correspond to the case where the pipe inlet was placed near the bottom or corner of the tank.

2. Nomenclature

$C_{p}$ : pressure coefficient

$C_{p 0}^{\prime}$ : pressure coefficient obtained by extrapolation of linear pressure distribution in pipe at $x=0$

$D$ : pipe inner diameter

$E$ : sum of energy

$H$ : obstacle height

$L$ : obstacle position

$p:$ pressure

$p_{0}:$ pressure at the same level as pipe axis in the reservoir

Re : Reynolds number

$r$ : radial coordinate

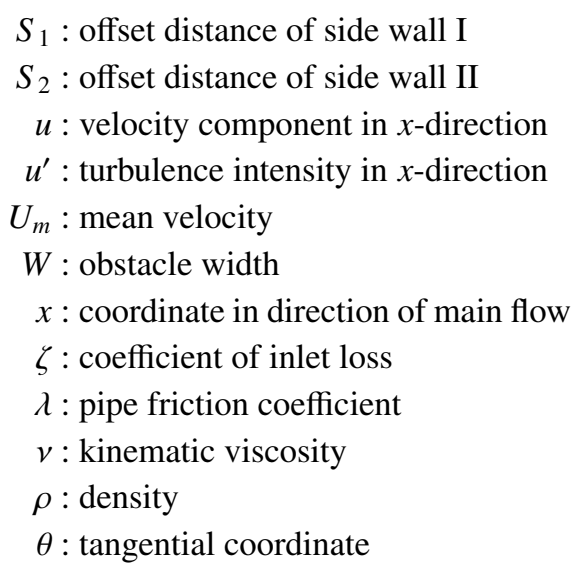

\section{Experimental Apparatus and Procedure}

\subsection{Experimental Apparatus}

The experimental setup is shown in Fig.3. The water in the reservoir (1) $(750 \times 800 \times$ $1000 \mathrm{~mm}$ ) is pumped into the test section (2) (inner diameter $D=30[\mathrm{~mm}]$ ), which is made of transparent acrylic resin and positioned horizontally, by the pump (5). After the water passes through the test section it comes back to the reservoir. The flow rate is measured with a flowmeter (4) and a controlled valve and bypass (7). To measure the pressure distribution along the pipe, many pressure taps with a diameter of $0.6[\mathrm{~mm}]$ placed at the mid-height level of the pipe and a reversed U-shape manometer 8 were used. The velocity and turbulence intensity distributions in the $x$-direction were measured using a laser Doppler anemometer, LDA (ID. Flow visualization was carried out using a water solution of fluorescent sodium dye and a laser light sheet ((13), (14). The visualized flow pattern was recorded with a digital video camera (16). 


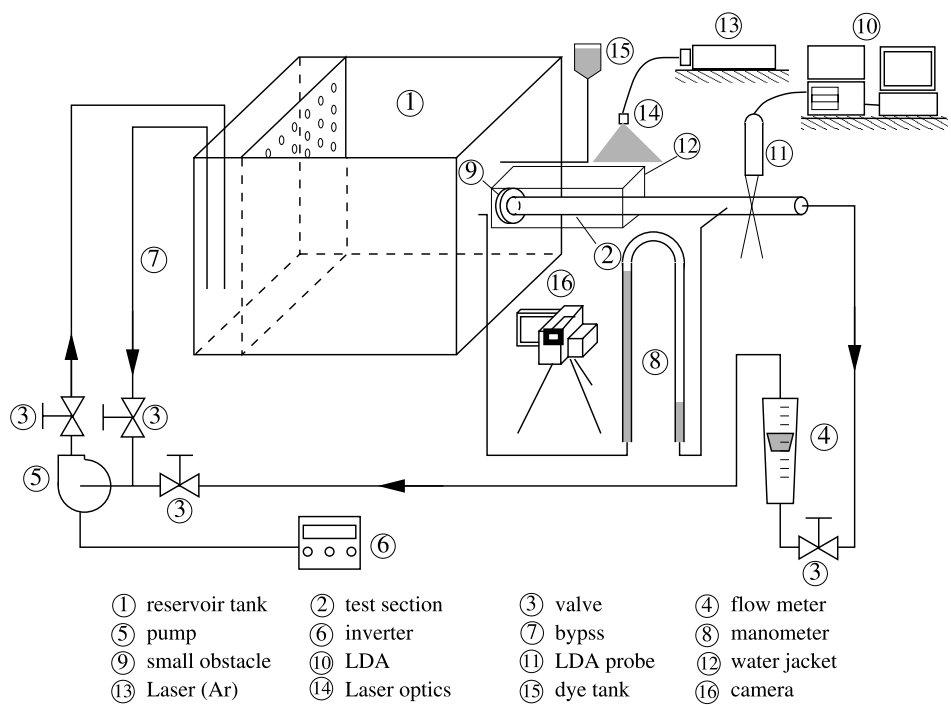

Fig. 3 Experimental setup

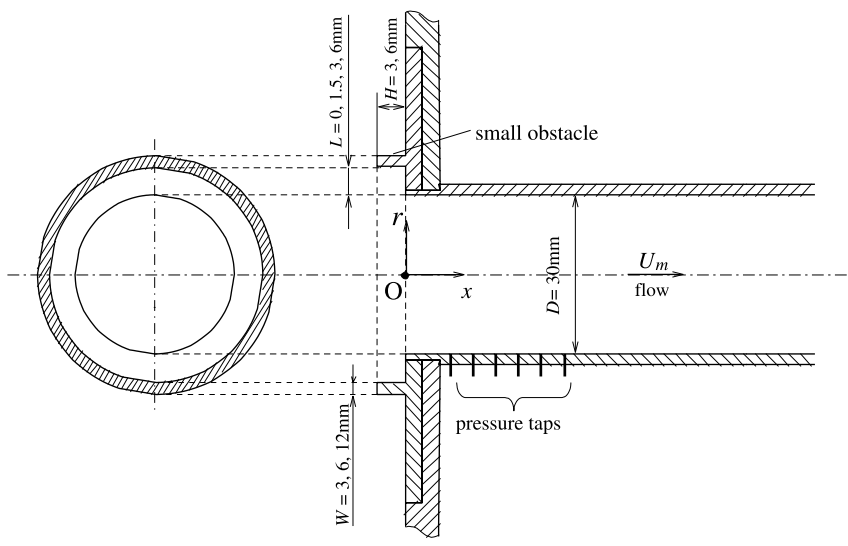

Fig. 4 Test section
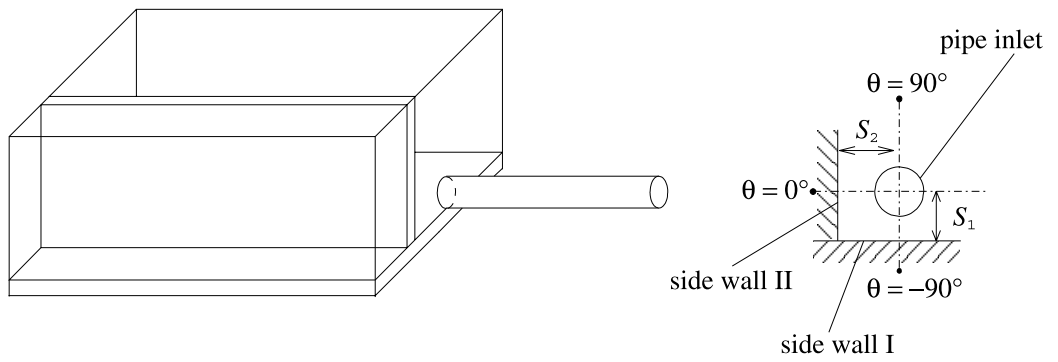

Fig. 5 Side walls and their offset distances

The mean velocity $U_{m}$ and diameter of the pipe $D$ were used as a reference velocity and length, and the experiment was carried out mainly for Reynolds number $\operatorname{Re}=U_{m} D / v=5.0 \times 10^{4}$.

Figure 4 illustrates the details of the test section and coordinate system. There are many pressure taps on the pipe wall at the mid-height level of the pipe. To control the formation of the vortex region at the pipe inlet, a small ring-shaped obstacle was mounted on the wall of the reservoir. We examined obstacles that had measured heights of $H / D=0.1$ and 0.2 , and widths of $W / D=0.1$ to 0.4 . The obstacle position $L / D$ varied from 0 to 0.2 .

The effects of the offset distances were also examined. Here, $S_{1} / D$ and $S_{2} / D$ are the sidewall distances, and they varied from $0.5 D$ to $3 D$ [see Fig.5]. In the experiment with the offset wall, the pressure distributions on the pipe wall for the top (at $\theta=90^{\circ}$ ) and the bottom (at $\theta=-90^{\circ}$ ) were measured as well as the mid-height level (at $\theta=0^{\circ}$ ). 


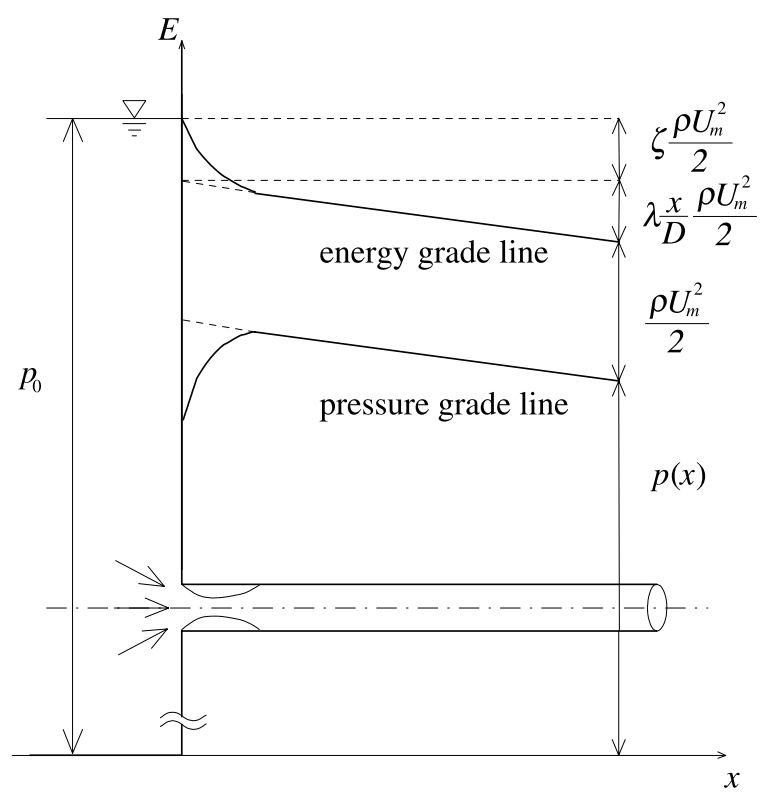

Fig. 6 Definition of $\zeta$

\subsection{Inlet loss or drag}

Figure 6 depicts the energy distribution $E$ along the pipe axis. The energy conservation between the reservoir and the pipe is expressed by

$p_{0}=\frac{\rho U_{m}^{2}}{2}+p(x)+\lambda \frac{x}{D} \frac{\rho U_{m}^{2}}{2}+\zeta \frac{\rho U_{m}^{2}}{2}$

where $p_{0}$ is the pressure at the same level as the pipe axis in the reservoir.

If we divide both sides by $\rho U_{m}^{2} / 2, \zeta$ can be expressed by

$$
\zeta=-\left\{\frac{2\left[p(x)-p_{0}\right]}{\rho U_{m}^{2}}+1+\lambda \frac{x}{D}\right\}
$$

The first term on the right hand side is pressure coefficient $C_{p}$, which can be expressed by the following equation in the fully developed region:

$$
C_{p}(x)=-\lambda x / D+C_{p 0}^{\prime}
$$

where $C_{p 0}^{\prime}$ is the constant in the reservoir.

Generally, the pressure gradient in the fully developed region corresponds to the value given by the Blasius resistance formula:

$$
\frac{d C_{p}}{d(x / D)}=-\lambda=-0.3164 \mathrm{Re}^{-\frac{1}{4}}
$$

Here, $\lambda$ is calculated from experimental results. The drag coefficient (nondimensional inlet loss) is calculated by

$$
\zeta=-\left[C_{p 0}^{\prime}+1\right]
$$

\section{Results and Discussion}

\subsection{Normal pipe inlet}

To find the optimum shape of $W \times H$ and mounting position $L$ of a small obstacle, $W, H$ and $L$ were varied. The pressure and velocity distributions were measured at $\operatorname{Re}=U_{m} D / v=$ $5 \times 10^{4}$. For flow visualization, $\operatorname{Re}=3.0 \times 10^{3}$. 
4.1.1. Flow visualization Figures 7(a) and (b) show the visualized flow pattern for the cases without and with the small obstacle of $H / D=0.2, L / D=0.1$ and $W / D=0.1$, respectively. In the case without the small obstacle, it is observed that vortex is shed from the inlet corner.

On the other hand, when a small obstacle was mounted on the wall, water from the reservoir tank flowed smoothly along the pipe wall. This indicates that the turbulence intensity near the wall was reduced by mounting the small obstacle. The figure shows that a separated flow from the inner corner of the obstacle flowed into the pipe along the pipe wall, and then the vortex region is suppressed.

4.1.2. Velocity and turbulence intensity

(a) Velocity distribution Figure 8 plots a comparison of the velocity distribution $u$ between cases without and with a small obstacle with the dimensions $H / D=0.2$ and $W / D=0.1$ at $L / D=0.1$. With an obstacle, the velocity $u$ near the pipe wall is larger than the case without an obstacle at $x / D=2.5$ and 3.5, and the profile of $u$ is flatter than the case without an obstacle. According to Sec.3.1.1, we can assume that the separated flow from the small obstacle flows along the pipe wall, and then a vortex region at $x / D<2.5$ (pipe inlet) in a time-averaged flow field is suppressed by the small obstacle. At $x / D>10$, those profiles are almost the same.

(b) Turbulence intensity distribution Figure 9 shows the distribution of turbulence intensity $\overline{u^{\prime}} / U_{m}$. The turbulence intensity $\overline{u^{\prime}} / U_{m}$ without a small obstacle takes a large value at $r / D>0.25$, especially near the pipe wall. This is caused by a vortex region at $x / D>2.5$. On the other hand, the turbulence intensity with an obstacle is smaller. It seems that the vortex region is smaller or is suppressed by the small obstacle. At $x / D>10$, both distributions are almost the same.

In this study, it was not possible to measure the cross section of $x / D<2.5$ because the
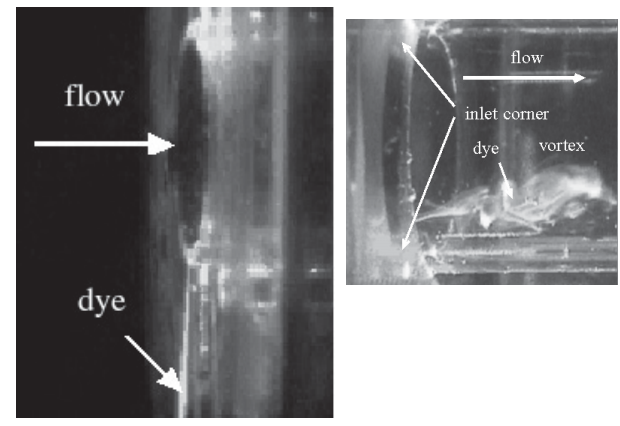

(a) without small obstacle

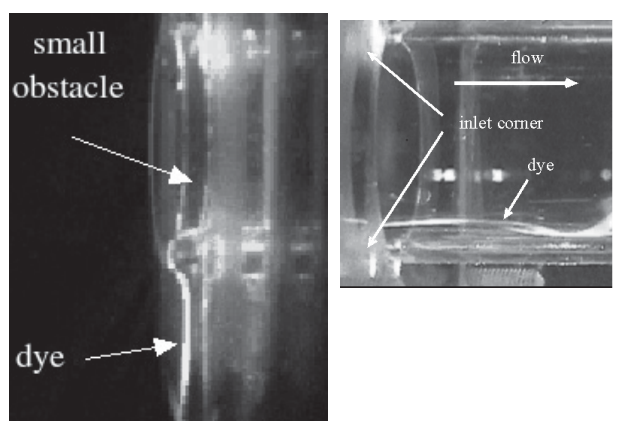

(b) with small obstacle

Fig. 7 Visualized flow pattern $\left(\operatorname{Re}=3 \times 10^{3}\right)$

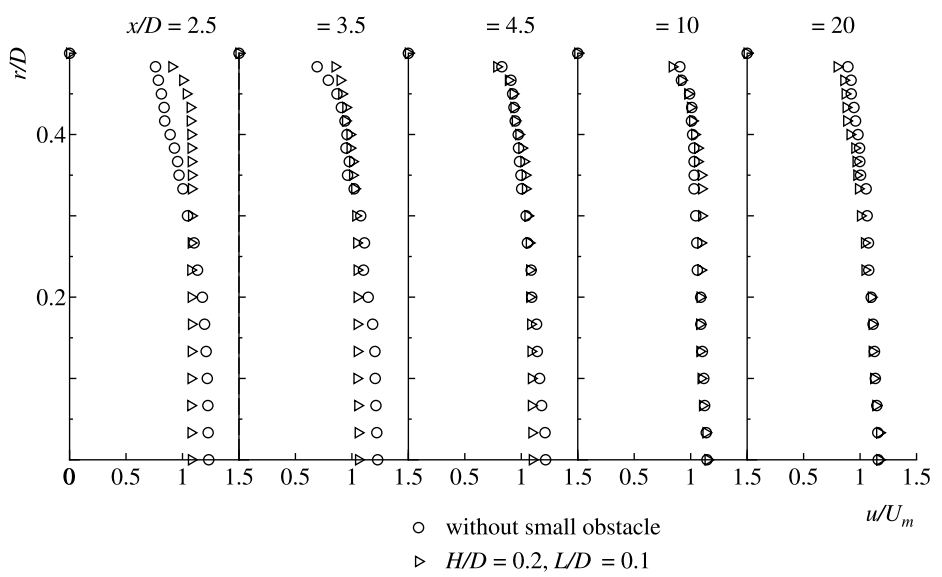

Fig. 8 Velocity distribution $\left(\operatorname{Re}=5 \times 10^{4}\right)$ 
LDA probe was too close to the reservoir wall. Therefore, it was not clarified whether it was possible to control a vortex region in a time-averaged flow pattern at the pipe inlet by mounting a small obstacle. However, we were able to determine that the vortex that was shed from the inlet corner could be suppressed by the small obstacle.

The vortex region in a time-averaged flow pattern on the pipe wall near the inlet can be minimized or suppressed by the small obstacle, and the effective cross-sectional area of the flow in the pipe can subsequently be increased. As a result, the inlet loss or drag, which is caused by negative pressure in the vortex region, is reduced.

\subsubsection{Pressure distribution}

(a) Without small obstacle The mark $\times$ in Fig.10 indicates the pressure distribution along the pipe wall without an obstacle. The pressure in the portion of the pipe after the inlet has a low value due to the vortex region and then increases rapidly as the flow moves downstream, reaching a maximal value at $x / D \simeq 2$. Finally, the pressure decreases with a constant gradient $d C_{p} / d(x / D)=0.210$, which depends only on friction loss. In this test section and with the value of $\operatorname{Re}=5.0 \times 10^{4}$, the pressure gradient by the Blasius resistance formula Eq.(4) is 0.212 , and it agrees well with the experimental results. The drag coefficient is $\zeta=0.388$.

(b) With small obstacle The pressure distribution along the pipe is shown in Figs.10 and 11 for $H / D=0.2$ and 0.1 , respectively. The obstacle width $W / D=0.1$ in both cases. In all cases with the small obstacle except $L / D=0.0$, the pressure recovery is faster and higher than the case without the obstacle. Especially, in $L / D=0.05,0.10$, low pressure in the area just after the inlet was not observed. As mentioned above, it appears that the change in the status of the vortex region causes the fast pressure recovery in the case with the small obstacle. When

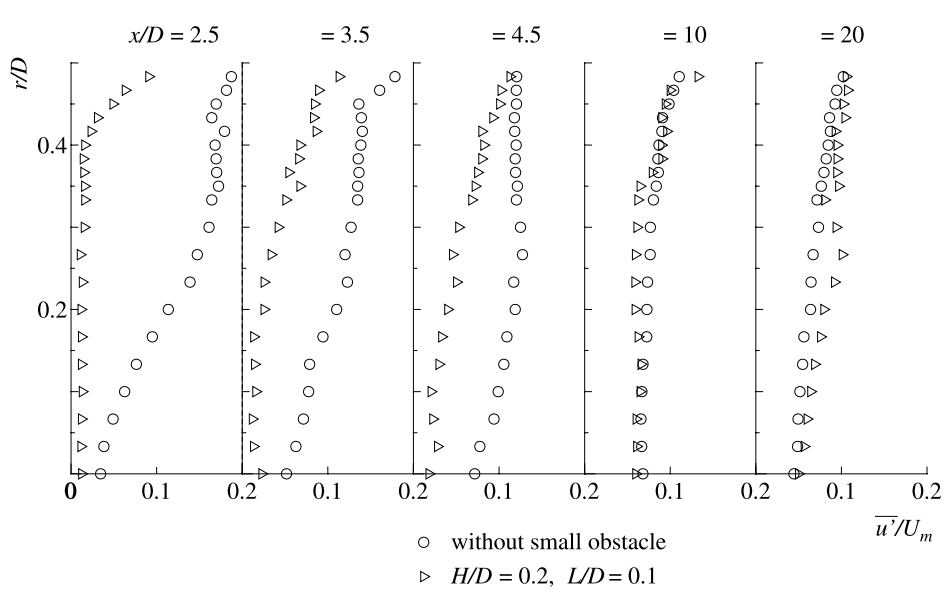

Fig. 9 Turbulence intensity distribution $\left(\operatorname{Re}=5 \times 10^{4}\right)$

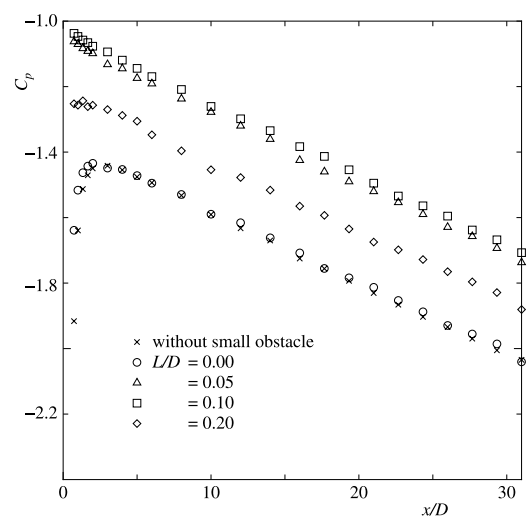

Fig. 10 Pressure distribution $(H / D=0.2, W / D=0.1)$

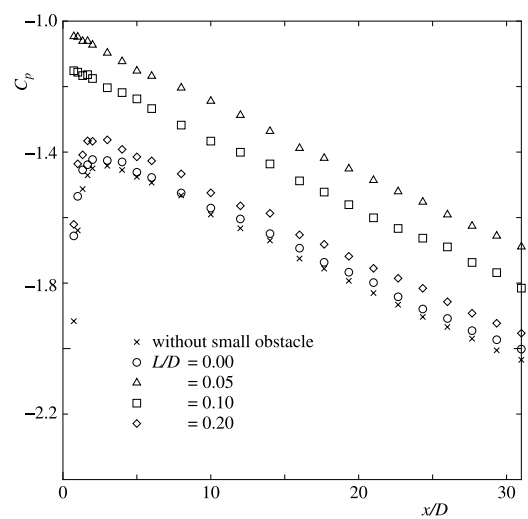

Fig. 11 Pressure distribution $(H / D=0.1, W / D=0.1)$ 
$L / D=0.0$, which corresponds to the case of the pipe protruding from the reservoir wall, the pressure distribution is almost the same as in the case without the small obstacle.

The pressure gradient of a linear distribution in the downstream portion was not affected by the small obstacle and had almost the same value as that obtained from Blasius's formula (4).

For $H / D=0.1$ and 0.2 , the pressure distribution had the highest values at $L / D=0.05$ and 0.10 , respectively.

4.1.4. Drag Reduction by Small Obstacle Figure 12 plots drag coefficient $\zeta$, or inlet loss, calculated by the pressure distribution mentioned before and given in Eq.(5). For comparison, $\zeta=0.388$ for the case without a small obstacle is also shown by the dashed-dotted line in Fig.12. The drag coefficient $\zeta$ has minimal values of 0.0364 and 0.0560 for the respective obstacle heights $H / D$ of 0.2 and 0.1 . The optimal obstacle positions $L / D$ that give the smallest $\zeta$ value are 0.1 and 0.05 for obstacle heights $H / D=0.2$ and 0.1 , respectively. The inlet loss, or drag, is reduced by about 85 to $90 \%$ in those cases. That position $L / D$ increases as the obstacle height $H / D$ increases, and $L / H$ is almost constant of 0.5 .

Figure 13 shows the effects of obstacle width $W / D$ on the drag coefficient $\zeta$. The obstacle height and position of the obstacle are $H / D=0.2$ and $L / D=0.1$. The pipe inlet loss, or drag, is not affected by the obstacle width $W / D$. The pipe inlet loss does not seem to be affected by the position of the outer corner of the small obstacle, but by the inner corner.

\subsection{Effects of side walls}

Next, we examined the effects of offset distance of a single side wall and two side walls (Fig.5) on the inlet loss or drag. With a single side (bottom) wall, we measured the pressure distribution for three different offset distances $S_{1}=0.5,1.0$, and 3.0. With two side (side and bottom) walls, we fixed the offset distance of side wall I at $S_{1} / D=0.5$ and changed the offset distances of side wall II so that $S_{2} / D=0.5,1.0$, or 3.0.

When the offset distance was $0.5 D$, the wall was tangential at the middle height or at the bottom of the circle, which corresponded to the pipe inlet corner, and the outside of the tangential line of the obstacle was cut since the obstacle interfered with the side wall.

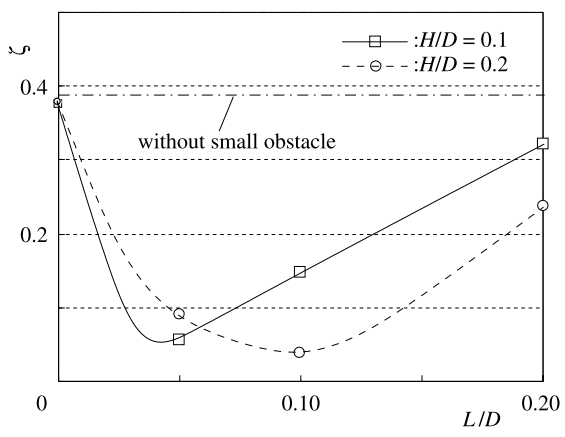

Fig. 12 Inlet loss $(W / D=0.1)$
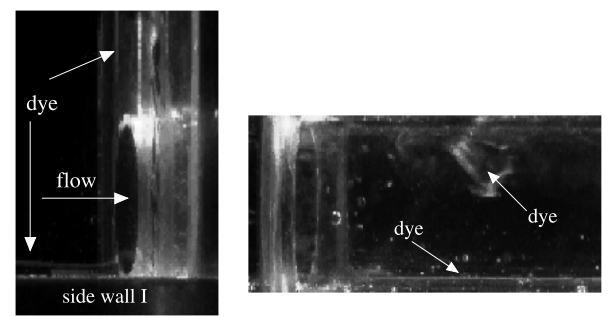

(a) Without small obstacle

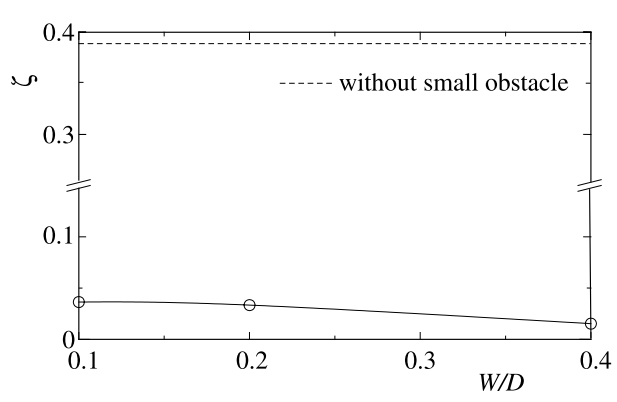

Fig. 13 Effects of obstacle width on inlet loss $(H / D=0.2, L / D=0.1)$
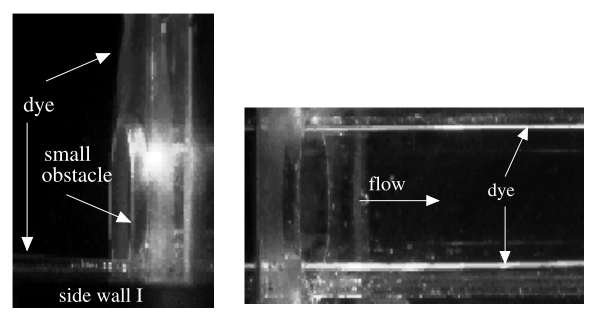

(b) $H / D=0.2, L / D=0.1, W / D=0.1$

Fig. 14 Visualized flow pattern $\left(S_{1} / D=0.5, \operatorname{Re}=3 \times 10^{3}\right)$ 
4.2.1. Flow visualization (single side plate) Figures 14(a) and (b) show the visualized flow pattern for the cases without and with the small ring-shaped obstacle in which $H / D=0.2$, $L / D=0.1$, and $W / D=0.1$. The offset distance was $S_{1} / D=0.5$, and Reynolds number was $\mathrm{Re}=3,000$ for both cases.

In the case without the obstacle [Fig.14(a)], the dye flowing from a small tap located on the upper part of the tank wall $\left(\theta=90^{\circ}\right)$ separated from the wall at the inlet corner and flowed into the pipe, forming a vortex region. On the other hand, dye coming out of a small tap on the tank bottom (side wall $\mathrm{I}, \theta=-90^{\circ}$ ) flowed along side wall $\mathrm{I}$ and the pipe wall without separation, and no vortex region was observed. Therefore, the flow pattern near the pipe inlet was not axisymmetric, and a vortex region existed on the upper side of the pipe but not near side wall I.

In the case with the obstacle, no vortex region of the upper side, which was observed in the case without the obstacle, was observed.

4.2.2. Velocity and turbulence intensity Figures 15(a) and (b) show the velocity $u$ and turbulence intensity $\overline{u^{\prime}}$ distribution for the same conditions as in 14(a) and (b), except the Reynolds number $\operatorname{Re}=5.0 \times 10^{4}$. We measured these distributions in the plane perpendicular to side wall I (bottom wall) and passing through the pipe axis. The distributions are asymmetric to the pipe axis especially in the case without the ring-shaped obstacle. The position of maximum velocity moves from the pipe axis toward side wall I. The velocity distribution near the side wall is flat, whereas the velocity side is reduced quickly as the flow approaches the pipe wall on the opposite side. The turbulence intensity near the side wall is much smaller than at the opposite side. It seems that the vortex region on the pipe wall near the side wall becomes smaller or is suppressed because the direction of flow near side wall I is originally in the same direction as the pipe wall.

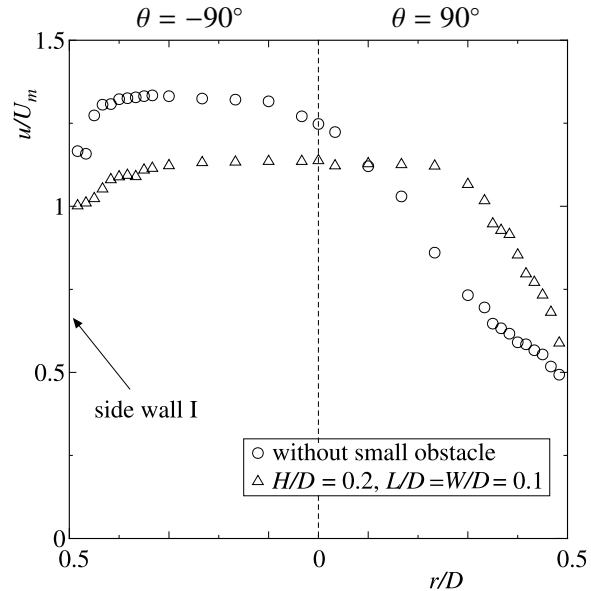

(a) Velocity distribution

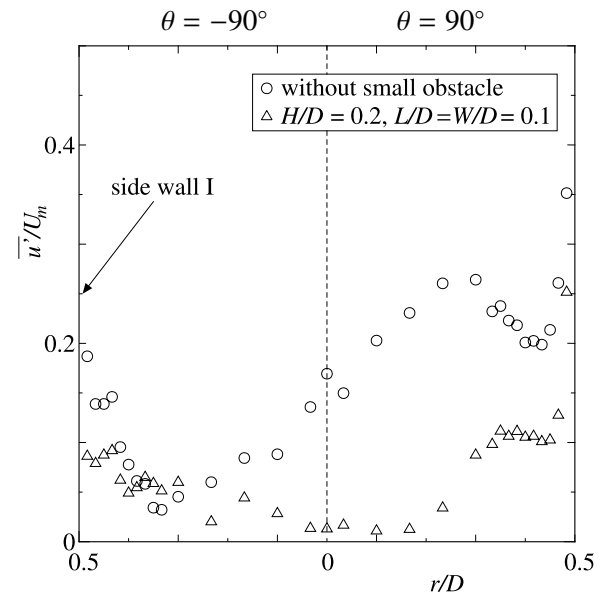

(b) Turbulence intensity distribution

Fig. 15 Velocity and turbulence intensity distribution

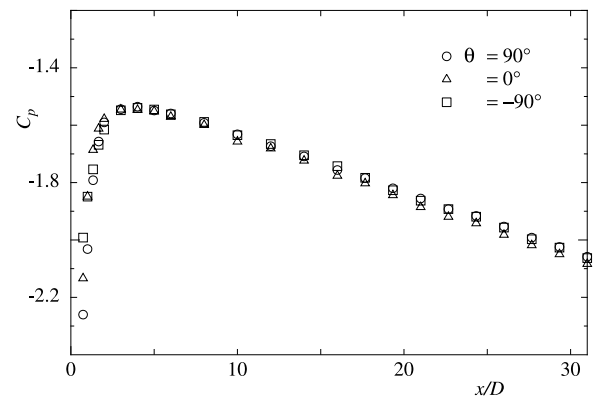

(a) $S_{1} / D=0.5$

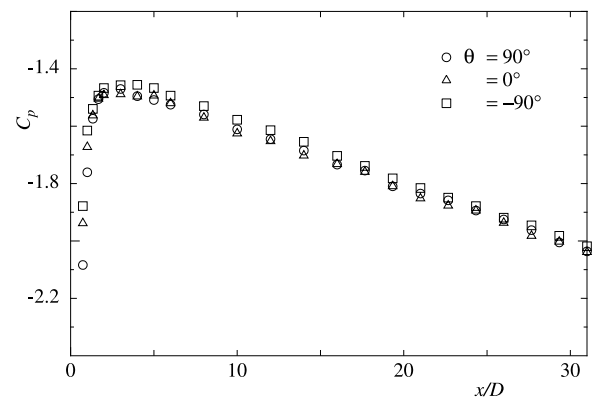

(b) $S_{1} / D=1.0$

Fig. 16 Effects of side wall I on pressure distribution (without small obstacle) 


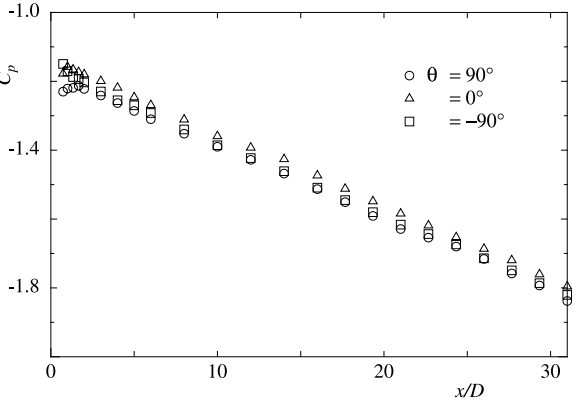

(a) $S_{1} / D=0.5$

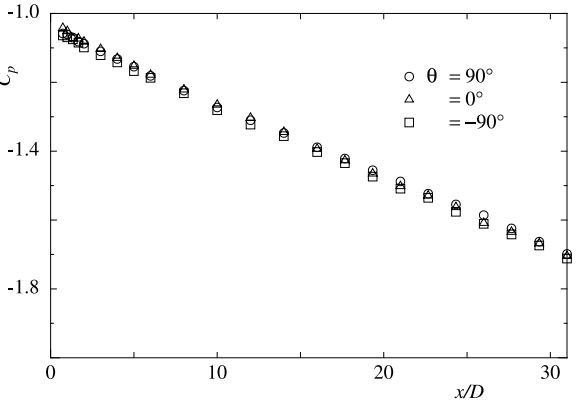

(b) $S_{1} / D=1.0$

Fig. 17 Effects of side wall I on pressure distribution $(H / D=0.2, W / D=0.1$ and $L / D=0.10)$

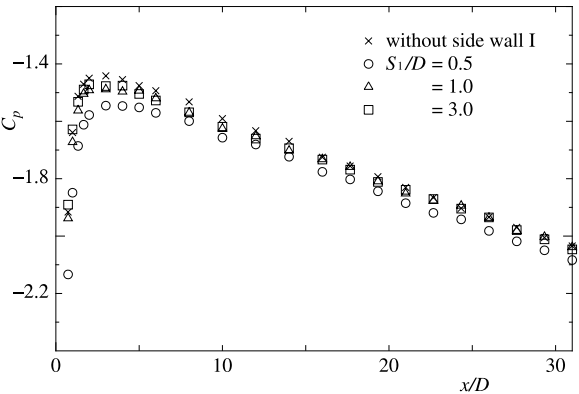

(a) without small obstacle

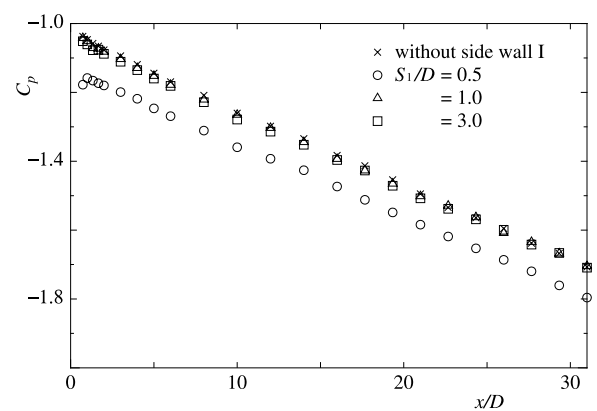

(b) $H / D=0.2, W / D=0.1$ and $L / D=0.10$

Fig. 18 Effects of offset distance of wall I on pressure distribution

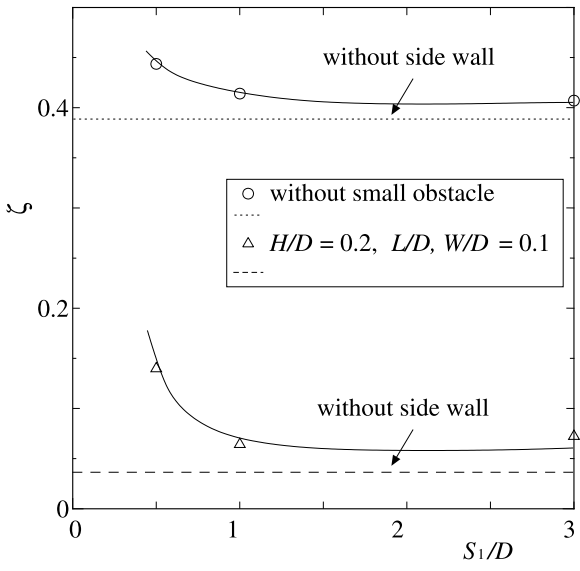

Fig. 19 Effects of small obstacle on inlet loss (side wall I)

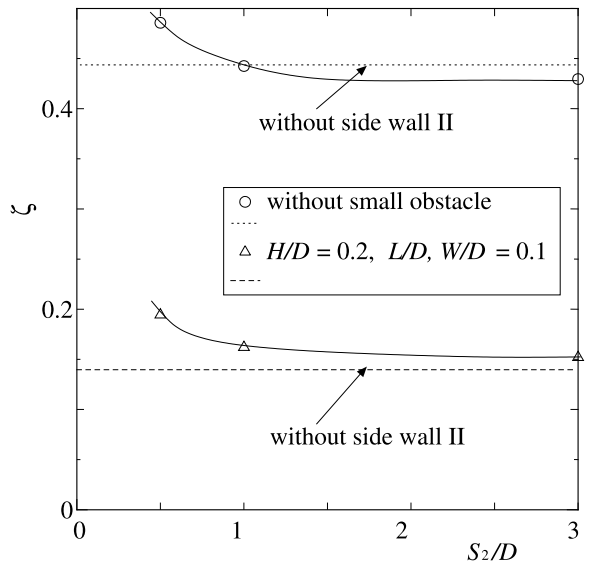

Fig. 20 Effects of small obstacle on inlet loss

(side walls I and II, $S_{1} / D=0.5$ )

\subsubsection{Pressure distribution and inlet loss}

(a) Pressure distribution The pressure distributions at $\theta=90^{\circ}, 0^{\circ}$, and $-90^{\circ}$ were measured. The results are plotted in Fig.16 (a) and (b) for $S_{1} / D=0.5$ and 1.0, respectively, without the small obstacle. Pressure distribution does not change for the angle $\theta$ except near the pipe inlet region. In this region, the pressure decreases as the angle of side wall I increases. This difference in pressure distribution seems to correspond to where the vortex region is smaller or suppressed near side wall I.

Figure 17 shows the effect of the small obstacle on pressure distribution. For $S_{1} / D=0.5$, there is a small pressure reduction near the pipe inlet at $\theta=-90^{\circ}$, but for $S_{1} / D=1.0$ it is hardly observed.

(b) Effects of offset distance of side wall Figures 18 (a) and (b) show the pressure distribu- 


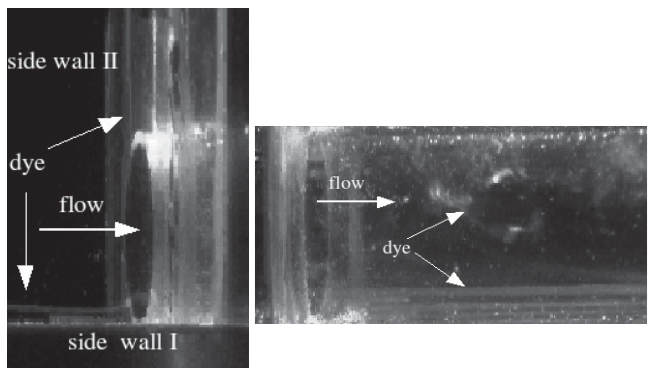

(a) without small obstacle
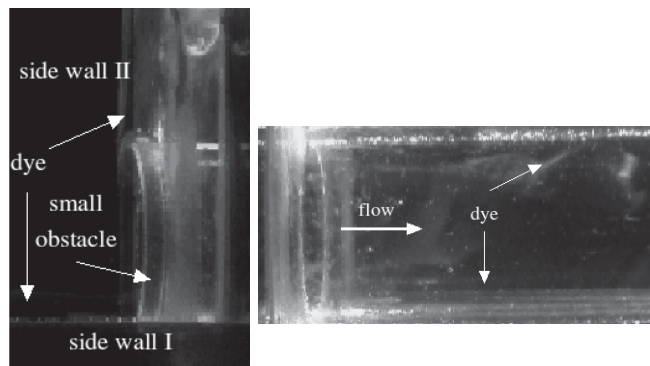

(b) with small obstacle

Fig. 21 Visualized flow pattern $\left(S_{1} / D=S_{2} / D=0.5, \operatorname{Re}=3 \times 10^{3}\right)$

tion without and with a small obstacle of $H / D=0.2$ and $L / D=0.1$ for $W / D=0.1$. The offset distance of side wall I is $S_{1} / D=0.5$ to 3.0 , and $\theta=0^{\circ}$.

All pressure distribution values in the developed region without the obstacle are almost the same except $S_{1} / D=0.5$. For this, the pressure is lower than other values in the entire region of $x$. The difference in pressure distributions with and without side wall I decreases as $S_{1} / D$ increases.

The pressure with a small obstacle becomes lower than without the small obstacle for each $S_{1} / D$.

(c) Inlet loss Figure 19 shows the effects a small obstacle has on inlet loss or drag $\zeta$. The inlet loss or drag is larger than the case without side wall I, except $S_{1} / D=0.5$ for both with and without a small obstacle. With incleasing $S_{1} / D$, the inlet loss decreases and approaches to the value for the case without side wall I. The inlet loss decreases by about 68,85 , and 82 percent for $S_{1} / D=0.5,1.0$, and 3.0 by mounting small obstacle, respectively.

4.2.4. Two side walls Figure 20 shows the effects a small obstacle has on inlet loss or $\operatorname{drag} \zeta$ for two side walls. For every case with side wall II, the inlet loss is larger than without side wall II, and the inlet loss decreases and approaches to the value for the case without the side wall I. The inlet loss respectively decreases by about 60,63 , and 64 percent for $S_{1} / D=0.5,1.0$, and 3.0, and these reduction rates are smaller than the case for the side wall I. It is because vortex shed from the corner at the opposite side of side wall I is not suppressed completely by a small obstacle as sown in the visualized flow pattern for $S_{1} / D=S_{2}=0.5$ of Fig.21.

\section{Conclusion}

We examined the degree of drag reduction of a pipe inlet flow by controlling and suppressing a vortex region using a small ring-shaped obstacle mounted on the reservoir wall. Some key results are as follows:

(1) The inlet loss (drag) coefficient $\zeta$ can be reduced by a maximum of about 90 percent by using a ring-shaped obstacle of height $H / D=0.2$ and at the position $L / D=0.1$. The obstacle width $W / D$ did not affect the drag reduction.

(2) Turbulence intensity near the pipe wall just after the inlet was reduced by the small obstacle. Additionally, we found that it was possible to minimize or suppress the vortex region after the inlet by using the obstacle.

( 3 ) With a single side wall, the inlet loss or drag became larger than without a side wall. However, the inlet loss was decreased when the offset distance of the side wall was increased. For $S_{1} / D=0.5$, the drag was about 10 percent larger than the larger offset distance, and it was reduced by about 68 percent using the small obstacle.

(4) With two side walls, the inlet loss or drag also became larger than without the second side wall. However, the inlet loss was reduced when the offset distance of the second side wall was increased and was almost the same as the value without the second side wall for $S_{2} / D>1.0$. Thus, drag was reduced by the small obstacle by about 60 percent. 


\section{References}

( 1 ) Tropea, C. D. and Gackstatter, R., The Flow Over Two-Dimensional Surface-Mounted Obstacle at Low Reynolds Numbers, Trans. ASME, J. Fluids Eng., 107-4, pp. 489-494, 1985.

( 2 ) Kiya, M. and Nozawa, T., Turbulence Structure in the Leading Edge Separation Zone of a Blunt Circular Cylinder, Trans. JSME, 53-488B, pp. 1183-1189, 1986.

( 3 ) Taulbee, D. B. and Robertson, J. M., Turbulent Separation Analysis Ahead of a Step, Trans. ASME, J. Basic Engineering, 94-3, pp. 544-550, 1972.

( 4 ) Liou, T.-M. and Kao, C.-F., Symmetric and Asymmetric Turbulent Flows in a Rectangular Duct With a Pair of Applied Mechanics, Trans. ASME, J. Fluids Engineering, 110, pp.505-508, 1963.

( 5 ) Chiang, T. P., Bifurcations of Flow Through Plane Symmetric Channel Contraction, Trans. ASME, J. Fluids Engineering, 424, pp. 444-451, 2002.

( 6 ) Fukunishi, Y., Kobayashi, R., Kikuchi, S., and Taniguchi, H., Active Control of a Separation-Reattachment Flow after a Forward Facing Step, Trans. JSME, 63-605B, pp. 76-81, 1997.

( 7 ) Ando T., Shakouchi, T., and Nakano, K., Flow Characteristics over Forward Facing Step and Control of Separated Flow, Trans. JSME, 65-637B, pp. 3008-3014, 1999.

( 8 ) Ando, T., Shakouchi, T., and Miyata, K., Drag Reduction of Abrupt Contraction Pipe (Control of Flow Separation by Small Obstacle), Trans. JSME, 70-691B, pp. 650-656, 2004. 